\title{
Mutational characteristics of bone metastasis of lung cancer
}

\author{
Xin Huang ${ }^{1}$, Xiaoliang Shi ${ }^{2}$, Donghua Huang ${ }^{1}$, Binghao Li ${ }^{1}$, Nong Lin ${ }^{1}$, Weibo Pan ${ }^{1}$, Xiaobo Yan ${ }^{1}$, \\ Hengyuan $\mathrm{Li}^{1}$, Qing $\mathrm{Hao}^{2}$, Zhaoming $\mathrm{Ye}^{1}$
}

${ }^{1}$ Department of Orthopedics, The Second Affiliated Hospital of Zhejiang University School of Medicine, Hangzhou, China; ${ }^{2}$ OrigiMed Co. Ltd., Shanghai, China

Contributions: (I) Conception and design: Z Ye; (II) Administrative support: Z Ye, X Huang; (III) Provision of study materials or patients: X Huang, Z Ye, D Huang, B Li, N Lin, W Pan, X Yan, H Li; (IV) Collection and assembly of data: X Huang, X Shi, Q Hao, N Lin, W Pan, X Yan; (V) Data analysis and interpretation: X Huang, X Shi, Q Hao, D Huang, B Li; (VI) Manuscript writing: All authors; (VII) Final approval of manuscript: All authors.

Correspondence to: Zhaoming Ye. Department of Orthopedics, The Second Affiliated Hospital of Zhejiang University School of Medicine, No. 1511 Jianghong Road, Hangzhou 310052, China. Email: yezhaoming@zju.edu.cn.

Background Roughly $30-40 \%$ of lung cancer (LC) patients develop bone metastasis during the course of disease. The genetic differences between primary LC and matched bone metastasis are not yet fully understood.

Methods: A total of 40 LC patients with bone metastasis were collected and 450 targeted cancer-related genes were sequenced for genomic-alteration (GA) identification.

Results: Among the 40 LC patients, 33 had adenocarcinomas and 7 had squamous cell carcinomas. The metastatic sites of the 33 lung adenocarcinomas (LUADs) were the pelvis (6 patients), spine (16 patients), and limbs (11 patients). A total of 425 and 422 GAs were detected in the primary and metastatic lesions, respectively. The most common GAs were epidermal growth factor receptor (EGFR) mutations, which had mutation rates of $85.0 \%$ and $72.5 \%$ in the primary and metastatic lesions, respectively, and tumor protein 53 (TP53) mutations, which had mutation rates of $52.5 \%$ and $67.5 \%$ in the primary and metastatic lesions, respectively. Metastases to the pelvis and spine were most commonly accompanied by factor receptor substrate 2 (FRS2), cyclin-dependent kinase 4 (CDK4), and murine double minute 2 (MDM2) amplification, and cyclindependent kinase inbibitor $2 A(C D K N 2 A)$ deletion. The concordance between primary lung squamous cell carcinoma (LUSC) and corresponding metastasis was significantly higher than that of primary LUAD and corresponding metastasis $(\mathrm{P}=0.033)$. Compared to limb and pelvis metastases, the shared mutation in spine metastasis was significantly lower $(\mathrm{P}=0.016$ and $\mathrm{P}=0.023$, respectively). In matched primary LUSCs and bone metastasis lesions, there was no significant difference in the distribution of the tumor mutational burden $(\mathrm{TMB})(\mathrm{P}=0.9)$. Conversely, a significant difference of the TMB distribution was detected in pairs of primary LUAD and corresponding bone metastasis lesions $(\mathrm{P}=0.021)$.

Conclusions: The consistency of mutation patterns between primary LC lesions and matched bone metastases may vary in terms of metastatic sites, but is very high in general. There was a significant difference in the TMB between primary LUAD and matched bone metastatic lesions. Our findings contribute to molecular understandings of primary LC and matched bone metastatic lesions.

Keywords: Bone metastasis; lung cancer (LC); next generation sequencing (NGS); genomic alterations; tumor mutational burden

Submitted May 25, 2021. Accepted for publication Jul 13, 2021.

doi: 10.21037/apm-21-1595

View this article at: https://dx.doi.org/10.21037/apm-21-1595 


\section{Introduction}

Lung cancer (LC) is one of the most common cancers in the world (1). LC patients are prone to bone metastasis, which may be accompanied by bone pain, fracture, hypercalcemia, nerve compression and other bone-related events that seriously affect patients' quality of life $(2,3)$. Once a tumor metastasizes to the bone, it is almost incurable and has a high mortality rate (4). However, the early detection of LC is still challenging, and most patients have bone metastasis at first diagnosis (5).

The incidence of bone metastasis in LC is about $22.3 \%$, which is related to the location and pathological type of the primary cancer $(6,7)$. Routine diagnostic methods include biopsy, imaging, and laboratory examination. The most common sites of bone metastasis of LC is the spine, which accounts for nearly $50 \%$ of bone metastasis (8). The application of next-generation sequencing (NGS) technology is widely used in the auxiliary diagnosis and target therapy of cancers $(9,10)$. In recent years, with the rapid development of precision medicine in LC, the survival of patients with advanced LC has improved significantly (11). Epidermal growth factor receptor (EGFR) is the most widely used driving gene for the targeted treatment of LC, and responds well to EGFR tyrosine kinase inhibitors (12). Kondraciuk et al. reported the genomic characters and examined risk factors for prognosis of thyroid cancer bone metastasis (13). Previous studies have shown that micro RNA, Dickkopf1, and insulin-like growth factor binding protein 3 are potential therapeutic targets for LC bone metastasis (14).

There is heterogeneity between primary lung and bone metastases. Previous studies have shown that bone metastasis of LC may be related to mutations in primary LC. Taverna et al. reported that interactions between the EGFR gene pathway and bone microenvironment affect the bone metastasis of LC (15). The mutation of the Kirsten rat sarcoma viral oncogene homolog (KRAS) gene may be associated with the postoperative recurrence and metastasis of LC (16). Echinoderm microtubule-associated protein-like 4 anaplastic lymphoma kinase (EML4-ALK) fusion is common in LC patients with distant organ metastasis, which suggests that the $A L K$ gene fusion is involved in the bone metastasis of LC (17-19). Mukai et al. reported the high expression of mesenchyma-to-epithelial transition (MET) in both primary and bone metastasis of LC patients (20), and suggested that drugs targeted at MET amplification, such as crizotinib and cabotinib, would have a certain effect on LC patients with bone metastasis.
Many reports have focused on the molecular mediators of non-small-cell lung carcinoma bone metastasis; however, its underlying mechanism remains unclear. In this study, we aimed to analyze the molecular differences between the primary LC and the matched bone metastases and provide evidence for the precise treatment of bone metastasis of LC. We present the following article in accordance with the MDAR reporting checklist (available at https://dx.doi. org/10.21037/apm-21-1595).

\section{Methods}

\section{Patient enrollment and sample collection}

A total of $40 \mathrm{LC}$ patients with bone metastasis at The Second Affiliated Hospital of Zhejiang University School of Medicine were enrolled in this study. Formalin-fixed paraffin-embedded (FFPE) tumor tissues from both primary and bone metastatic lesions were collected and transferred to OrigiMed, Shanghai for genetic variation detection. Matched blood samples were also collected as a control. Genomic deoxyribonucleic acid (DNA) was prepared using the QIAamp DNA FFPE Tissue Kit and the QIAamp DNA Blood Midi Kit (Qiagen, Hilden, Germany) in accordance with the manufacturer's instructions. The concentration of DNA was measured by Qubit and normalized to $20-50 \mathrm{ng} / \mu \mathrm{L}$. The study was conducted in accordance with the Declaration of Helsinki (as revised in 2013). The study was approved by the Ethical Committee of The Second Affiliated Hospital of Zhejiang University School of Medicine (No. 2020143), and informed consent was provided by all patients.

\section{Identification of GAs and the TMB}

The genomic mutations were identified using the NGSbased YuanSu450 gene panel (OrigiMed, Shanghai, China), which covers the coding exons of 450 cancerrelated genes and 64 selected introns in 39 genes that are frequently rearranged in solid tumors. The genes were captured and sequenced with a mean depth of $800 \times$ using the Illumina NextSeq 500. Genomic alterations (GAs), including single nucleotide variants (SNVs), insertiondeletion polymorphisms (Indels), copy number variation, were identified using MuTect (v.1.17), PINDEL (v.2.04), and Control-FREEC (v9.4), respectively. Gene fusions were detected through an in-house pipeline, and gene rearrangements were assessed by the Integrative Genomics 
Table 1 Clinicopathologic features of the cohort

\begin{tabular}{lc}
\hline Variable & $\mathrm{n}(\%)$ \\
\hline Total & 40 \\
Gender & $25(62.5)$ \\
Male & $15(37.5)$ \\
Female & $63[45-83]$ \\
Age, median [range] & \\
Smoking status & $18(45.0)$ \\
Smoking & $22(55.0)$ \\
Nonsmoking & \\
TMB, median [range] & $3.7[0.6-41.4]$ \\
Primary & $3.1[0-0.7]$ \\
Metastases & \\
Primary subtype & $33(82.5)$ \\
Lung adenocarcinoma & $7(17.5)$ \\
Lung squamous cell carcinoma & $17(42.5)$ \\
Bone metastases loci & $160.0)$ \\
Pelvis & \\
Spine & \\
\hline
\end{tabular}

TMB, tumor mutational burden.

Viewer (IGV). The tumor mutational burden (TMB) was estimated by counting the somatic mutations, including SNVs and Indels, per mega base of the sequence examined in each patient. Driver mutations and known germline alterations were not counted.

\section{Statistical analysis}

The statistical analyses were performed using SPSS version 22.0 (SPSS Inc., Chicago, IL, USA). Fisher's exact test was used for the significant differences analyses. A $\mathrm{P}<0.05$ was considered statistically significant.

\section{Results}

\section{Patient characteristics}

Forty LC patients with bone metastases were enrolled in this study. Among them, there were 25 males and 15 females, $18(45.0 \%)$ patients had a history of smoking for
$>20$ years and $22(55.0 \%)$ never smoked. Patients had a median age of 63 years (range, $45-83$ years). According to the primary subtype, $33(82.5 \%)$ patients had lung adenocarcinoma (LUAD) and $7(17.5 \%)$ had lung squamous cell carcinoma (LUSC). The bone metastasis sites of this cohort included 7 (17.5\%) pelvis, 17 (42.5\%) spine, and 16 (40.0\%) limb sites (see Table 1).

\section{Different characterization of $G A$ s between primary $L C$ and bone metastasis}

A total of 604 clinically relevant GAs were evaluated in this cohort (see Figure 1). The mutational characteristics of LUSC and LUAD were different. For example, the amplifications of mitogen-activated protein kinase 13 (MAP3K13), phosphatidylinositol 3-kinase catalytic alpha polypeptide (PIK3CA), B-cell lymphoma 6 (BCL6), E26 transformation-specific variant transcription factor 5 (ETV5), and Kelch-like protein 6 (KLHL6) were more frequent in LUSC than LUAD (see Figure 1). Only 2 patients had the KRAS G12V mutation for which the corresponding primary tumor was LUAD.

In primary tissues, the most common mutated genes included EGFR (62.5\%, 25/40), tumor protein 53 (TP53) (50.0\%, 20/40), cyclin-dependent kinase inbibitor $2 \mathrm{~A}$ (CDKN2A), bigh mobility group protein 2 (HMGA2), and murine double minute 2 (MDM2) (15.0\%, 6/40, for each), and mucin 16 (MUC16), cyclin-dependent kinase 4 (CDK4), $v$-myc avian myelocytomatosis viral oncogene homolog (MYC), protein kinase C iota (PRKCI), and Tetraspanin 31 (TSPAN31) (12.5\%, $5 / 40$, for each). The bone metastasis samples from 39 patients were successfully sequenced, and the most common mutated genes include EGFR and TP53 (64.1\%, 25/39, for both), PRKCI (17.9\%, 7/39), factor receptor substrate 2 (FRS2), MAP3K13, and PIK3CA (15.4\%, 6/39, for each), and BCL6, CDKN2A, CDKN2B, ETV5, KLHL6, MUC16, SRY-Box Transcription Factor 2 (SOX2), and telomerase ribonucleic acid component (TERC) $(12.8 \%, 5 / 39$, for each). In this cohort, 235 (40.2\%, 235/585) GAs were shared in paired primary lesions and bone metastases, 165 $(28.2 \%, 165 / 585)$ GAs were specifically detected in primary lesions, and 185 (31.6\%, 185/585) GAs were specifically detected in bone metastases. However, no mutation was associated with bone metastases and primary tumors.

\section{Association between mutated genes and metastatic locations in LUAD}

Given the heterogeneity between LUSC and LUAD 


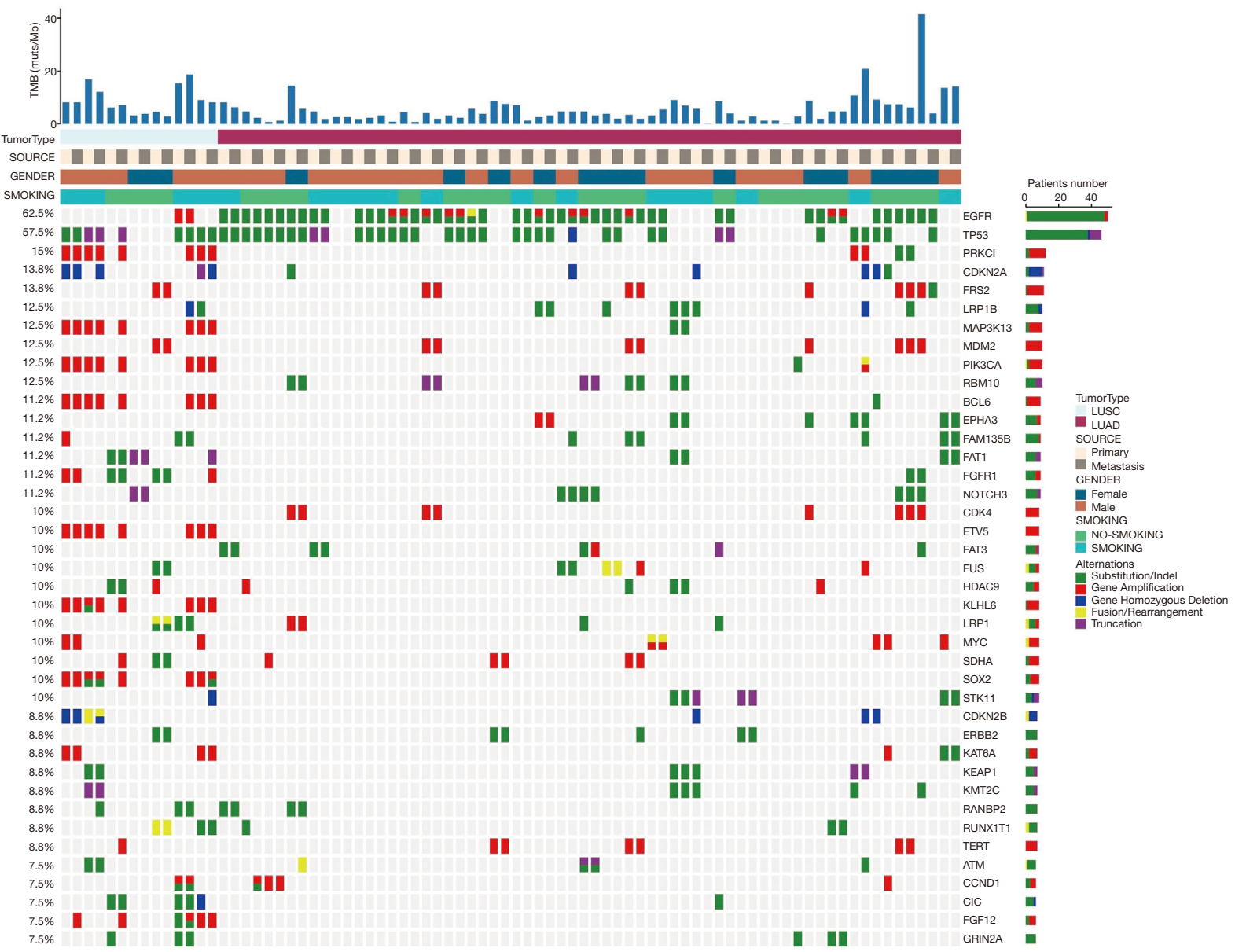

Figure 1 Mutational landscape of paired primary tumors and bone metastases of $40 \mathrm{LC}$ patients. The X-axis shows each case sample, and the Y-axis shows each mutated gene. The upper bar graph shows the TMB values of the patients. The bar graph on the right shows the number of each mutated gene. Tumor type: blue represents LUSC and red represents LUAD; Source: light yellow represents primary tumors and grey represents bone metastases; Gender, blue represents female and brown represents male; Smoking: green represents no-smoking and blue represents smoking; Alterations: green represents substitution/indel mutations, red represents gene amplification mutations, blue represents gene homozygous deletion mutations, yellow represents fusion/rearrangement mutations, and purple represents truncation mutations. LC, lung cancer; TMB, tumor mutational burden; LUSC, lung squamous cell carcinoma; LUAD, lung adenocarcinoma.

and the small number of samples, we selected 33 patients with primary LUAD tumors to further investigate the molecular characterizations of different metastatic loci. The mutational profiling of LUAD showed a higher frequency of FRS2, CDK4, and MDM2 amplification, and the homozygous deletion of $C D K N 2 A$ in patients with pelvis and spine metastases, but not in those with the limb metastases (see Figure 2). Based on the primary and metastatic mutational profile of each patient, the mean frequency of shared mutations of patients with limb, pelvic, and spine metastases were $63.1 \%$ (range, 0.0-91.7\%),
58.4\% (range, $44.4-80.0 \%$ ), and $38.7 \%$ (range, $5.26-$ $80.0 \%$ ), respectively. A statistical analysis showed that the frequency of shared mutations in the spine metastases was significantly lower than the frequency in the limbs and the pelvis $(\mathrm{P}=0.016$ and $\mathrm{P}=0.023$, respectively; see Figure 3$)$.

\section{Decreased TMB in LUAD with bone metastasis}

To explore the relationship between the TMB and metastatic loci, available TMB values were measured from both primary and metastatic samples. The median TMB 

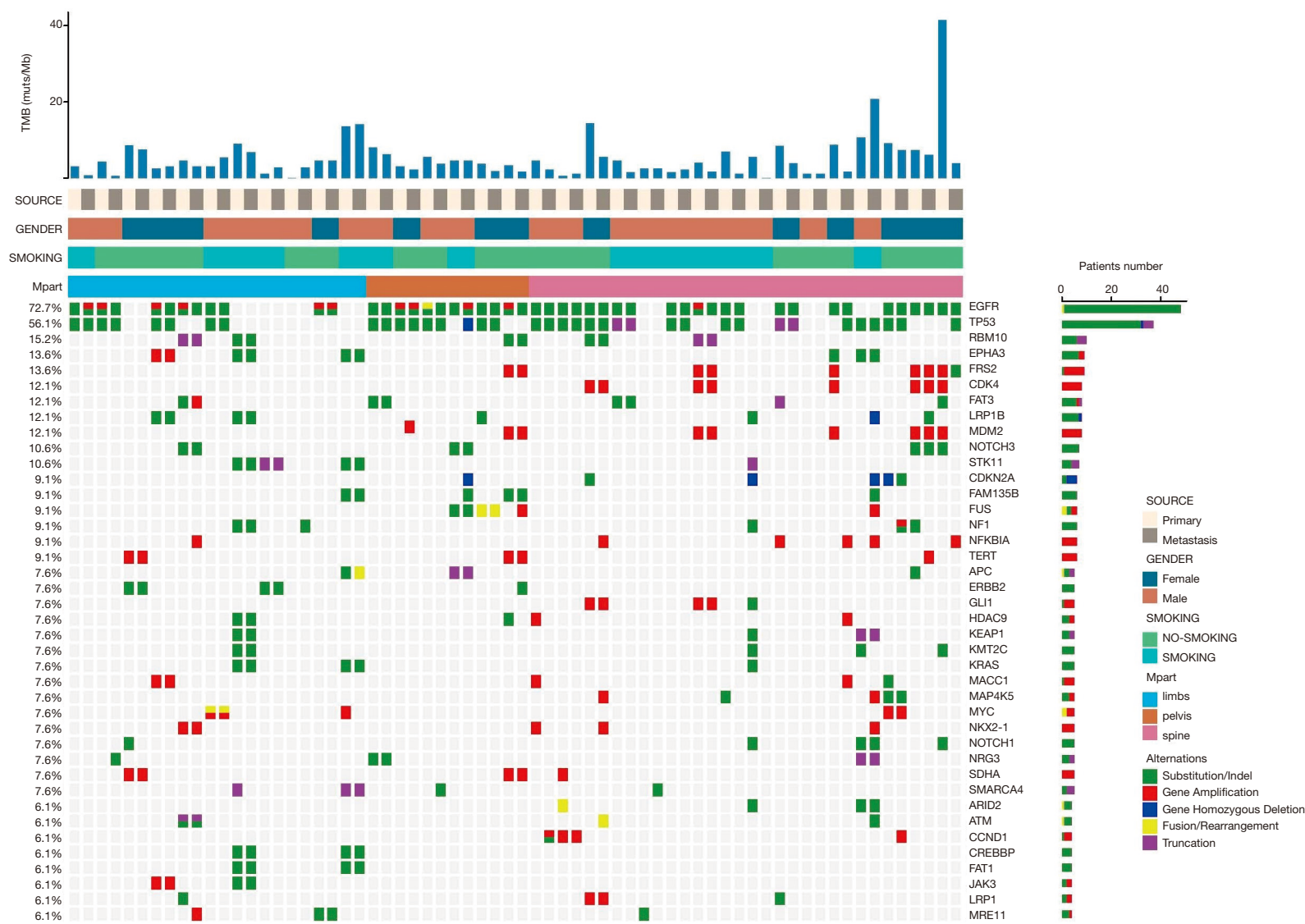

Figure 2 Mutational landscape of paired primary tumor and bone metastases of 33 LUAD patients. The X-axis shows each case sample, and the Y-axis shows each mutated gene. The upper bar graph shows the TMB value of the patients. The bar graph on the right shows the number of each mutated gene. Source: light yellow represents primary tumors and grey represents bone metastases. Gender, blue represents female and brown represents male. Smoking: green represents no-smoking and blue represents smoking. Mpart: blue represents limb metastasis, red represents pelvis metastasis, and pink represents spine metastasis. Alterations: green represents substitution/indel mutations, red represents gene amplification mutations, blue represents gene homozygous deletion mutations, yellow represents fusion/rearrangement mutations, and purple represents truncation mutations. LUAD, lung adenocarcinoma; TMB, tumor mutational burden.

values of primary and metastatic tumors were 3.7 and 3.1 muts $/ \mathrm{Mb}$, respectively. According to the subtypes of primary lesions, our results showed that the TMB value of bone metastasis was significantly lower than that of primary LUAD $(\mathrm{P}=0.021)$, but there was no significant difference in TMB values between bone metastasis and primary LUSC $(\mathrm{P}=0.9$; see Figure $4 A)$. Additionally, the TMB value of the bone metastasis of LUAD was also significantly lower than that of bone metastasis of LUSC $(\mathrm{P}=0.0098$; see Figure $4 A)$. We also compared the TMB value in different metastatic loci of LUAD, but no significant difference was found (see Figure 4B).

\section{Discussion}

Different subtypes of LC have preferential metastatic sites, such as liver metastasis of small cell LC, and brain metastasis of small cell LC and adenocarcinoma $(21,22)$. The percentage of bone metastases in LC patients ranges from $20 \%$ to $40 \%$ (23). Research on the epidemiological and prognostic factors of LC bone metastasis has progressed $(24,25)$; however, the molecular characterization of LC bone metastasis remains unclear. To evaluate the difference in molecular characteristics between primary LC and bone metastasis, we performed NGS detections on the primary 
and bone metastasis samples of 40 patients, which included 33 LUAD and 7 LUSC samples.

Heterogeneity widely exists in primary tumors and bone metastases. Krawczyk et al. reported that the mutation rate of EGFR in bone metastases was $75 \%$, but only $12.8 \%$ in primary tumors (26). EGFR mutations are common in bone

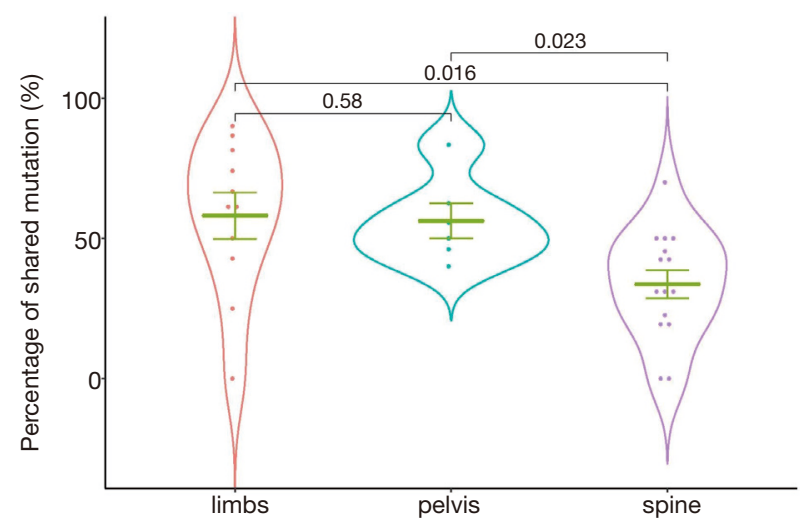

Figure 3 Comparison of the consistency of mutations between different metastasis sites and primary LUAD. The $\mathrm{X}$-axis represents the different metastasis sites, and the Y-axis represents the consistency of mutations for each patient. LUAD, lung adenocarcinoma. metastases of LUAD $(27,28)$. Guan et al. showed that the incidence of bone metastasis in patients with the EGFR mutation was higher than those with $E G F R$ wild-type mutation (29). In the present study, our results supported the wide heterogeneity of primary and metastatic lesions, and found only $40 \%$ of coexisting mutations. Among the mutations that were only detected in the primary tumor or corresponding metastasis of each patient, the EGFR mutation was still the most common mutation. The statistical analysis did not reveal any mutations associated with primary tumor or bone metastasis. This may be due to the small sample size of this cohort; future studies should be conducted with larger cohorts.

The KRAS gene is a factor of the mitogen-activated protein kinase (MAPK) pathway, which is downstream of the EGFR, and its mutation can promote cancer cell development (30). KRAS mutations commonly occur in about $30 \%$ of LUADs and $5 \%$ of LUSCs, and mutations in exon 12 are the most frequent (31). Different subtypes of $K R A S$ mutations are associated with different types of metastasis. Previous studies have shown that the KRAS G12C mutation occurs more frequently in western patients with developed bone metastasis, while the KRAS G12V mutation occurs more frequently in western patients with developed pleuro-pericardial metastasis $(32,33)$.
A

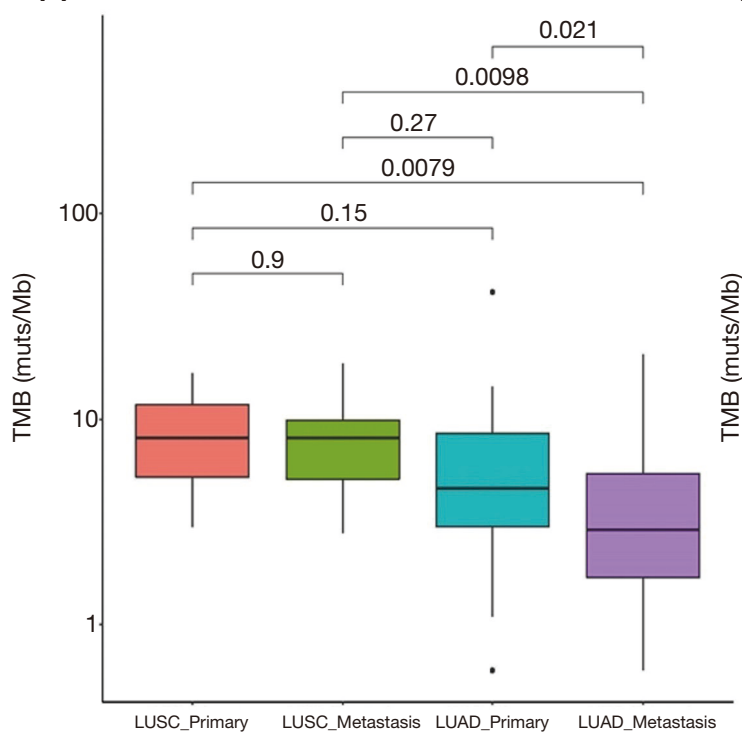

B

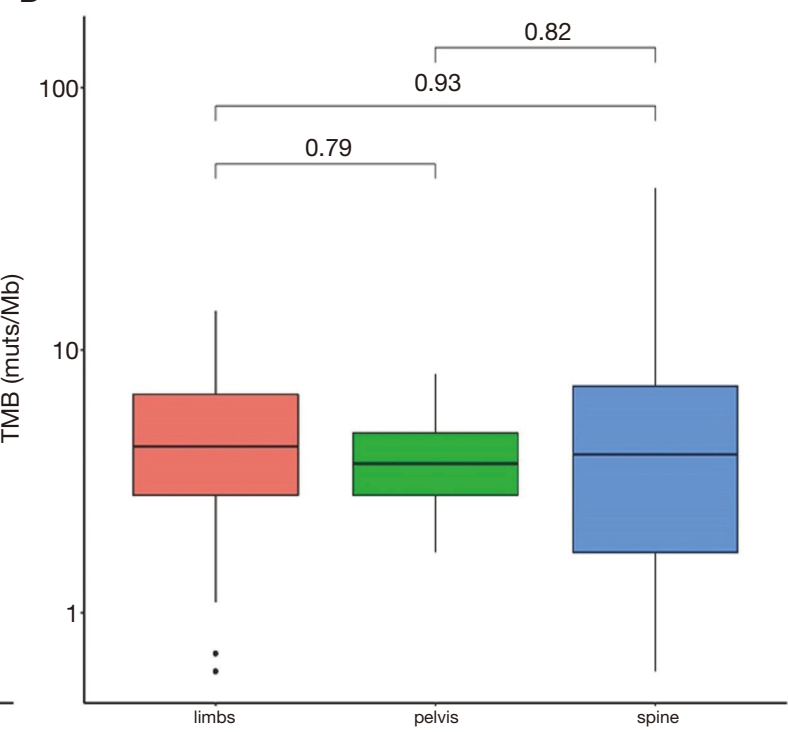

Figure 4 Distribution of TMB in this cohort. (A) Comparison of TMB value between the primary tumor and metastasis sites. (B) The distribution of TMB in different metastatic sites of LUAD. The $\mathrm{X}$-axis represents the different primary sites or bone metastatic sites, and the Y-axis represents the TMB value of each sample. TMB, tumor mutational burden; LUAD, lung adenocarcinoma. 
Interestingly, while all the samples in this cohort came from LC patients with bone metastasis, only 3 patients harbored KRAS G12V mutation, including a patient who failed to detect genomic detection of bone metastasis sample. This suggests that the KRAS mutation is not characteristic in Chinese LC patients with bone metastasis, and that there are different mechanisms between the Chinese and Western patient groups.

The TMB represents the number of somatic mutations per mega base of tumor DNA and may further guide the selection of checkpoint inhibitors for patients (34). A high TMB has been reported to be associated with the generation of neoantigens and potential clinical responses to immunotherapies (35). In a clinical LC trial, patients with a high TMB benefited significantly from immunotherapy, which improved progression-free survival and response rates (36). Given the heterogeneity of primary and metastatic tumors, we also compared the TMB value between primary and metastatic tumors. Interestingly, the TMB decreased significantly after bone metastasis in LUAD, but no significant TMB difference was found between primary LUSC and its bone metastasis. These results suggest that the benefits of immunotherapy may be affected if LUAD has metastasized to the bone.

Bone metastasis of LC commonly occurred in the spine and pelvis, followed by the limbs. Tani et al. reported that mesometastasis, which is defined as bone metastasis of the forearm or lower leg, might be a poor prognostic factor (37). A recent systematic meta-analysis identified several prognostic factors, including age, gender, disease factors, and increasing the time from diagnosis to surgery (38). In the present study, our results revealed that the metastatic spine had a higher heterogeneity than metastatic pelvis or limbs. The heterogeneity of a tumor may affect the outcome of a targeted therapy (39). We deduced that this heterogeneity may be a potential factor for the poor prognosis of metastatic spine. However, neither significant GAs nor significant different TMB were detected among the metastatic spine, pelvis, and limbs.

Recently, great progress has been made in targeted therapy such as EGFR tyrosine kinase inhibitors (TKIs) in lung cancer (40). The treatment of the bone metastasis of lung cancer is challenging. When the metastatic bone lesion was identified, the treatment of primary lung lesion and metastatic bone lesion should both be considered. However, the question of genetic heterogeneity between the primary tumor and the corresponding bone metastases is pivotal for the treatment. In this study, our results showed the high consistency of mutation patterns between primary LC lesions and matched bone metastases. This indicate that the effective treatment of primary lung cancer may be also suitable for matched bone metastasis of lung cancer, such as the EGFR-TKI treatment for the bone metastasis of lung cancer with sensitive EGFR mutations $(41,42)$.

In conclusion, this study identified the GAs of 40 pairs of primary LC and bone metastases, revealed the decreased TMB value of bone metastasis of LUAD, and the high heterogeneity between primary LUAD and the metastatic spine. Our results suggest that heterogeneity may be a potential factor for the poor prognosis of LC patients with bone metastasis, and immunotherapy should be used with caution among LUAD patients with bone metastasis. This study had a number of limitations, including that only a small number of patients participated in this study. In the future, a large cohort study needs to be conducted to examine the precise treatment of bone metastasis of LC.

\section{Acknowledgments}

The authors are grateful to all the study patient participants and their family members for their contributions and support.

Funding: This work was supported by the Zhejiang Provincial Natural Science Foundation of China (LY19H060007).

\section{Footnote}

Reporting Checklist: The authors have completed the MDAR reporting checklist. Available at https://dx.doi. org/10.21037/apm-21-1595

Data Sharing Statement: Available at https://dx.doi. org/10.21037/apm-21-1595

Conflicts of Interest: All authors have completed the ICMJE uniform disclosure form (available at https://dx.doi. org/10.21037/apm-21-1595). XS and QH report they are an employee of OrigiMed Co. Ltd. The other authors have no conflicts of interest to declare.

Ethical Statement: The authors are accountable for all aspects of the work, including ensuring that questions related to the accuracy or integrity of any part of the work have been appropriately investigated and resolved. The study was conducted in accordance with the Declaration of 
Helsinki (as revised in 2013). The study was approved by the Ethical Committee of The Second Affiliated Hospital of Zhejiang University School of Medicine (No. 2020143), and informed consent was provided by all patients.

Open Access Statement: This is an Open Access article distributed in accordance with the Creative Commons Attribution-NonCommercial-NoDerivs 4.0 International License (CC BY-NC-ND 4.0), which permits the noncommercial replication and distribution of the article with the strict proviso that no changes or edits are made and the original work is properly cited (including links to both the formal publication through the relevant DOI and the license). See: https://creativecommons.org/licenses/by-nc-nd/4.0/.

\section{References}

1. Torre LA, Bray F, Siegel RL, et al. Global cancer statistics, 2012. CA Cancer J Clin 2015;65:87-108.

2. Hernandez RK, Wade SW, Reich A, et al. Incidence of bone metastases in patients with solid tumors: analysis of oncology electronic medical records in the United States. BMC Cancer 2018;18:44.

3. da Silva GT, Bergmann A, Thuler LCS. Skeletal related events in patients with bone metastasis arising from nonsmall cell lung cancer. Support Care Cancer 2016;24:731-6.

4. Roodman GD. Mechanisms of bone metastasis. N Engl J Med 2004;350:1655-64.

5. Thunnissen E, Kerr KM, Herth FJ, et al. The challenge of NSCLC diagnosis and predictive analysis on small samples. Practical approach of a working group. Lung Cancer 2012;76:1-18.

6. da Silva GT, Bergmann A, Thuler LCS. Incidence and Risk Factors for Bone Metastasis in Non-Small Cell Lung Cancer Asian Pac J Cancer Prev 2019;20:45-51.

7. Li J, Zhu H, Sun L, et al. Prognostic value of site-specific metastases in lung cancer: A population based study. J Cancer 2019;10:3079-86.

8. Jya B. Differential derivation of momentum and energy equations in electroelasticity. Acta Mechanica Solida Sinica 2017;30:21-6.

9. Liu ZL, Wang C, Chen HJ, et al. Bone metastasis from lung cancer identified by genetic profiling. Oncol Lett 2017;13:847-50.

10. Szurian K, Kashofer K, Liegl-Atzwanger B. Role of NextGeneration Sequencing as a Diagnostic Tool for the Evaluation of Bone and Soft-Tissue Tumors. Pathobiology 2017;84:323-38.
11. Ruiz-Cordero R, Devine WP. Targeted Therapy and Checkpoint Immunotherapy in Lung Cancer. Surg Pathol Clin 2020;13:17-33.

12. Kauffmann-Guerrero D. Adjuvant TKI treatment of EGFR-mutant lung cancer-already ripe for decision? Transl Lung Cancer Res 2020;9:964-6.

13. Kondraciuk JD, Rice SL, Zhou X, et al. Thyroid Cancer Bone Metastasis: Survival and Genomic Characteristics of a Large Tertiary Care Cohort. Clin Nucl Med 2019;44:e465-71.

14. Pang $\mathrm{H}, \mathrm{Ma} \mathrm{N}$, Jiao $\mathrm{M}$, et al. The Biological Effects of Dickkopf1 on Small Cell Lung Cancer Cells and Bone Metastasis. Oncol Res 2017;25:35-42.

15. Taverna S, Pucci M, Giallombardo M, et al. Amphiregulin contained in NSCLC-exosomes induces osteoclast differentiation through the activation of EGFR pathway. Sci Rep 2017;7:3170.

16. Lohinai Z, Klikovits T, Moldvay J, et al. KRAS-mutation incidence and prognostic value are metastatic site-specific in lung adenocarcinoma: poor prognosis in patients with KRAS mutation and bone metastasis. Sci Rep 2017;7:39721.

17. Ou SH, Tan J, Yen Y, et al. ROS1 as a 'druggable' receptor tyrosine kinase: lessons learned from inhibiting the ALK pathway. Expert Rev Anticancer Ther 2012;12:447-56.

18. Kuijpers CCHJ, Hendriks LEL, Derks JL, et al. Association of molecular status and metastatic organs at diagnosis in patients with stage IV non-squamous nonsmall cell lung cancer. Lung Cancer 2018;121:76-81.

19. Nanjo $S$, Nakagawa T, Takeuchi $S$, et al. In vivo imaging models of bone and brain metastases and pleural carcinomatosis with a novel human EML4-ALK lung cancer cell line. Cancer Sci 2015;106:244-52.

20. Mukai S, Yorita K, Kawagoe Y, et al. Matriptase and MET are prominently expressed at the site of bone metastasis in renal cell carcinoma: immunohistochemical analysis. Hum Cell 2015;28:44-50.

21. Megyesfalvi Z, Tallosy B, Pipek O, et al. The landscape of small cell lung cancer metastases: Organ specificity and timing. Thorac Cancer 2021;12:914-23.

22. Hendriks LE, Smit EF, Vosse BA, et al. EGFR mutated non-small cell lung cancer patients: more prone to development of bone and brain metastases? Lung Cancer 2014;84:86-91.

23. Zarogoulidis K, Boutsikou E, Zarogoulidis P, et al. The impact of zoledronic acid therapy in survival of lung cancer patients with bone metastasis. Int J Cancer 2009;125:1705-9.

24. Zheng XQ, Huang JF, Lin JL, et al. Incidence, prognostic 
factors, and a nomogram of lung cancer with bone metastasis at initial diagnosis: a population-based study. Transl Lung Cancer Res 2019;8:367-79.

25. Sun JM, Ahn JS, Lee S, et al. Predictors of skeletal-related events in non-small cell lung cancer patients with bone metastases. Lung Cancer 2011;71:89-93.

26. Krawczyk P, Nicoś M, Ramlau R, et al. The incidence of EGFR-activating mutations in bone metastases of lung adenocarcinoma. Pathol Oncol Res 2014;20:107-12.

27. Confavreux CB, Girard N, Pialat JB, et al. Mutational profiling of bone metastases from lung adenocarcinoma: results of a prospective study (POUMOS-TEC). Bonekey Rep 2014;3:580.

28. Huang L, Jiang XL, Liang HB, et al. Genetic profiling of primary and secondary tumors from patients with lung adenocarcinoma and bone metastases reveals targeted therapy options. Mol Med 2020;26:88.

29. Guan J, Chen M, Xiao N, et al. EGFR mutations are associated with higher incidence of distant metastases and smaller tumor size in patients with non-small-cell lung cancer based on PET/CT scan. Med Oncol 2016;33:1.

30. Murray S, Dahabreh IJ, Linardou H, et al. Somatic mutations of the tyrosine kinase domain of epidermal growth factor receptor and tyrosine kinase inhibitor response to TKIs in non-small cell lung cancer: an analytical database. J Thorac Oncol 2008;3:832-9.

31. Garrido P, Olmedo ME, Gómez A, et al. Treating KRASmutant NSCLC: latest evidence and clinical consequences. Ther Adv Med Oncol 2017;9:589-97.

32. Renaud S, Seitlinger J, Falcoz PE, et al. Specific KRAS amino acid substitutions and EGFR mutations predict sitespecific recurrence and metastasis following non-small-cell lung cancer surgery. Br J Cancer 2016;115:346-53.

33. Renaud S, Falcoz PE, Schaëffer M, et al. Prognostic value

Cite this article as: Huang $\mathrm{X}$, Shi $\mathrm{X}$, Huang D, Li B, Lin N, Pan W, Yan X, Li H, Hao Q, Ye Z. Mutational characteristics of bone metastasis of lung cancer. Ann Palliat Med 2021;10(8):88188826. doi: 10.21037/apm-21-1595 of the KRAS G12V mutation in 841 surgically resected Caucasian lung adenocarcinoma cases. Br J Cancer 2015;113:1206-15.

34. Riaz N, Havel JJ, Makarov V, et al. Tumor and Microenvironment Evolution during Immunotherapy with Nivolumab. Cell 2017;171:934-949.e16.

35. Subudhi SK, Vence L, Zhao H, et al. Neoantigen responses, immune correlates, and favorable outcomes after ipilimumab treatment of patients with prostate cancer. Sci Transl Med 2020;12:eaaz3577.

36. Hellmann MD, Ciuleanu TE, Pluzanski A, et al. Nivolumab plus Ipilimumab in Lung Cancer with a High Tumor Mutational Burden. N Engl J Med 2018;378:2093-104.

37. Tani S, Morizaki Y, Uehara K, et al. Bone metastasis of limb segments: Is mesometastasis another poor prognostic factor of cancer patients? Jpn J Clin Oncol 2020;50:688-92.

38. Gao ZY, Zhang T, Zhang H, et al. Prognostic factors for overall survival in patients with spinal metastasis secondary to prostate cancer: a systematic review and meta-analysis. BMC Musculoskelet Disord 2020;21:388.

39. Bedard PL, Hansen AR, Ratain MJ, et al. Tumour heterogeneity in the clinic. Nature 2013;501:355-64.

40. Kim C, Liu SV. First-line EGFR TKI therapy in nonsmall-cell lung cancer: looking back before leaping forward. Ann Oncol 2019;30:1852-5.

41. Han C, Ma J, Zhao J, et al. EGFR mutations, gene amplification, and protein expression and KRAS mutations in primary and metastatic tumors of nonsmall cell lung cancers and their clinical implications: a meta-analysis. Cancer Invest 2011;29:626-34.

42. Wang S, Wang Z. Meta-analysis of epidermal growth factor receptor and KRAS gene status between primary and corresponding metastatic tumours of non-small cell lung cancer. Clin Oncol (R Coll Radiol) 2015;27:30-9. 